\title{
Padrões alimentares de idosos e seus determinantes: estudo de base populacional no sul do Brasil
}

\author{
Dietary patterns of elderly Brazilians and associated determinants: \\ a population-based study in the south of Brazil
}

Ana Paula Gomes (https://orcid.org/0000-0003-2842-7340) ${ }^{1}$

Isabel Oliveira Bierhals (https://orcid.org/0000-0002-8739-8669) ${ }^{1}$

Luna Strieder Vieira (https://orcid.org/0000-0001-9946-8575) ${ }^{1}$

Ana Luiza Gonçalves Soares (https://orcid.org/0000-0003-2763-4647) ${ }^{1}$

Thaynã Ramos Flores (https://orcid.org/0000-0003-0098-1681) ${ }^{1}$

Maria Cecília Formoso Assunção (https://orcid.org/0000-0002-7767-8835) ${ }^{1}$

Helen Gonçalves (https://orcid.org/0000-0001-6470-3352) ${ }^{1}$

${ }^{1}$ Programa de Pós-

Graduação em

Epidemiologia,

Universidade Federal

de Pelotas. R. Marechal

Deodoro $1160 / 3^{\circ}$,

Centro. 96020-220

Pelotas RS Brasil.

anapaulagomes.nutri@

gmail.com

\begin{abstract}
This cross-sectional study sought to assess the effect of demographic and socioeconomic factors, chronic diseases, and health counseling on dietary patterns of the elderly. Food consumption was assessed by means of a reduced eating frequency questionnaire distributed to 1,426 elderly residents in Pelotas, State of Rio Grande do Sul, Brazil, and dietary patterns were obtained using main component analysis. Two dietary patterns were identified: healthy diet (wholegrain food, fruits, vegetables, and milk) and Western diet (sweet snacks, fried foods, frozen foods, processed meats, and fast food). Females, non-smokers, and alcohol-free individuals tended more toward the healthy diet, whereas males, smokers, alcoholdependent, and overweight individuals tended more toward the Western diet. Higher income and schooling were associated with a higher tendency toward both diets, and chronic diseases were not associated with either diet. Receiving counseling for adopting healthy habits was associated with a higher tendency toward a healthy diet and a lower tendency toward the Western diet. There is a need to increase effective guidance on healthy habits by health services to promote healthier eating practices, with special attention to elderly males, smoke$r$ s, alcohol-dependent and overweight individuals. Key words Eating behavior, Eating habits, Elderly, Cross-sectional studies
\end{abstract}

Resumo Este estudo teve como objetivo avaliar o efeito de fatores demográficos e socioeconômicos, de doenças crônicas e do recebimento de orientações em saúde no padrão alimentar de idosos. $O$ consumo alimentar foi avaliado por meio de questionário de frequência alimentar reduzido aplicado a 1.426 idosos residentes em Pelotas, RS, Brasil e os padrões alimentares foram obtidos por análise de componentes principais. Dois padrões foram identificados: saudável (alimentos integrais, frutas, legumes/verduras e leite) e ocidental (doces, frituras, alimentos congelados, embutidos e "fast food"). Mulheres, não fumantes e não dependentes alcoólicos aderiam mais ao padrão saudável, enquanto que homens, fumantes, dependentes alcóolicos e indivíduos com sobrepeso aderiam mais ao padrão ocidental. Maior renda e escolaridade estiveram associadas à maior adesão de ambos os padrões e a presença de doenças crônicas não esteve associada a nenhum padrão. O recebimento de orientações para hábitos saudáveis esteve associado à maior adesão ao padrão saudável e menor ao padrão ocidental. Há necessidade do fortalecimento de orientações eficazes sobre hábitos saudáveis nos serviços de saúde, com especial atenção para idosos do sexo masculino, tabagistas, dependentes alcoólicos e indivíduos com sobrepeso.

Palavras-chave Comportamento alimentar, Hábitos alimentares, Idoso, Estudos transversais 


\section{Introdução}

Atualmente, $14,3 \%$ da população brasileira é constituída por idosos ( $\geq 60$ anos) e estima-se que esta proporção dobre nas próximas duas décadas $^{1,2}$. O crescimento acelerado da população idosa acarreta, de imediato, aumento da carga de doenças crônicas não transmissíveis (DCNT) ${ }^{3}$. Cerca de $80 \%$ dos idosos brasileiros têm pelo menos uma $\mathrm{DCNT}^{4}$, o que representa um grande desafio ao sistema de saúde, com a necessidade de maior ênfase e investimentos em ações de promoção e prevenção da saúde ${ }^{5}$.

A dieta inadequada é um importante fator de risco modificável para diversas $\mathrm{DCNT}^{6}$. Em razão disso, é crescente o interesse da literatura sobre o consumo dos grupos de alimentos que definem os padrões alimentares saudáveis e não saudáveis ${ }^{7}$. O padrão alimentar consiste no conjunto ou grupos de alimentos que são consumidos por uma determinada população ${ }^{8}$. Por expressarem os efeitos combinados e potencialmente sinérgicos dos diversos alimentos consumidos na dieta habitual de uma determinada população, a Organização Mundial da Saúde (OMS) recomenda que a avaliação do consumo alimentar em estudos populacionais seja baseada em padrões alimentares ${ }^{9}$.

Os padrões alimentares podem ser detectados por meio de índices dietéticos baseados em recomendações alimentares (abordagem a priori) ou por meio de métodos estatísticos de redução e/ou agregação de componentes (abordagem a posteriori $)^{10}$. Os padrões derivados por meio da abordagem a posteriori são específicos da população em estudo, podendo não se repetir em outras $^{10}$. Todavia, este método possui a vantagem de refletir o comportamento real de um grupo populacional, fornecendo informações úteis para a criação de diretrizes nutricionais ${ }^{10}$.

A escolha alimentar é influenciada tanto por determinantes individuais (conhecimento do indivíduo sobre alimentação e nutrição, suas percepções sobre alimentação saudável, idade e situação de saúde), como coletivos (fatores socioeconômicos como renda e escolaridade, fatores sociais e culturais $)^{11}$. Entre os idosos, além desses fatores, alterações anatômicas e funcionais que ocorrem em decorrência do processo de envelhecimento e a presença de DCNT também devem ser considerados, uma vez que repercutem diretamente na condição de saúde do indivíduo e no processo de escolha alimentar ${ }^{12}$.

A orientação sobre hábitos saudáveis realizada por profissionais de saúde constitui uma im- portante estratégia na promoção da alimentação saudável em adultos e idosos. Estudos nacionais e internacionais realizados com adultos e idosos têm demonstrado que indivíduos que receberam orientação para hábitos saudáveis possuem maior probabilidade de aderir a estes hábitos ${ }^{13-17}$.

Estudos avaliando o padrão alimentar de idosos ainda são escassos no Brasil ${ }^{10,18,19}$, além disso, fatores determinantes dos padrões alimentares em idosos, além dos demográficos e socioeconômicos, são pouco conhecidos e, até o momento, não foram encontrados estudos nacionais de base populacional que avaliassem o efeito das DCNT ou do recebimento de orientações sobre hábitos saudáveis nos padrões alimentares de idosos. Assim, o objetivo do estudo foi avaliar o efeito de fatores demográficos e socioeconômicos, de morbidades crônicas e do recebimento de orientações em saúde no padrão alimentar de idosos.

\section{Métodos}

Estudo transversal, de base populacional, realizado com a população idosa ( $\geq 60$ anos) residente na cidade de Pelotas, região Sul do Brasil. A coleta de dados ocorreu entre os meses de janeiro e agosto de 2014.

O processo amostral foi realizado em dois estágios. Primeiramente, todos os setores censitários da cidade de Pelotas, com base no Censo 2010, foram ordenados de acordo com a renda média e, após, os domicílios dentro de cada setor foram selecionados sistematicamente. Ao final do processo, 133 setores e 4.123 domicílios foram eleitos para a pesquisa. Foram elegíveis para o estudo os idosos não institucionalizados residentes na zona urbana de Pelotas. Foram localizados 1.839 idosos, dos quais 1.451 foram entrevistados. Destes, não foram elegíveis para este estudo, os idosos em nutrição enteral ou parenteral $(\mathrm{n}=5)$. Assim, a amostra final foi constituída por 1.426 idosos que apresentavam dados completos sobre alimentação. As perdas e as recusas totalizaram 10,8\% (n=199/1.839) e 11,6\% (n $=214 / 1.839$ ), respectivamente, sendo superiores no sexo feminino $(61,0 \%)$. A média de idade foi menor quando comparada à daqueles que constituíram a amostra final do estudo (69,5 anos e $70,7$ anos, respectivamente; $\mathrm{p}=0,010)$.

O consumo alimentar foi avaliado por meio de questionário reduzido de frequência alimentar referente à semana antecedente à entrevista, que foi desenvolvido para outro estudo ${ }^{12}$ tendo como base as principais recomendações de con- 
sumo alimentar contidas no Guia Alimentar para a população brasileira ${ }^{20,21}$. O questionário investigou o consumo de: (1) frutas, (2) legumes/ verduras, (3) alimentos integrais (como pão integral, arroz integral, aveia), (4) leite e derivados (iogurte, queijo), (5) carnes ou ovos, (6) frituras, (7) guloseimas (como doces, refrigerantes e sucos artificiais), (8) embutidos ou enlatados (como salsicha, presunto, sardinha em lata), (9) alimentos congelados prontos para consumo (como pizza, lasanha, hambúrguer, nuggets) e (10) fast food.

Os padrões alimentares foram identificados por meio de análise de componentes principais (ACP).Aplicou-se teste de esfericidade de Bartlett (BTS) e a medida de adequação da amostra Kaiser-Meyer-Olkin (KMO), para avaliar a aplicabilidade do método fatorial ao conjunto de dados. $\mathrm{O}$ valor de KMO foi de 0,660 e o de BTS $<0,001$, indicando bom ajuste da análise fatorial ${ }^{22}$. A rotação ortogonal varimax foi utilizada de forma a obter padrões não correlacionados entre si e melhorar a interpretação dos dados. O número de padrões a serem extraídos foi definido com base no gráfico scree plot, no qual os pontos de maior declive indicam o número de fatores a ser considerado na análise. $\mathrm{O}$ modelo foi construído fixando o número de padrões a serem retidos conforme o número indicado pelo gráfico, sendo identificados dois padrões alimentares. Os grupos que contribuíram para a caracterização de cada padrão foram aqueles com cargas fatoriais $\geq 0,3$ ou $\leq-0,3$. Os padrões foram nomeados com base nas características dos itens retidos em cada um. As variáveis foram divididas em tercis, sendo o maior tercil indicativo de maior adesão ao padrão.

As variáveis independentes utilizadas foram:

Demográficas e socioeconômicas: sexo; idade (60-69, 70-79 e 80 anos ou mais); escolaridade (em anos completos: 0-3, 4-7, 8-11 e 12 ou mais) e renda familiar (categorizada em quintis);

Relacionadas à saúde e comportamentos: tabagismo (consumo de pelo menos 1 cigarro ao dia, há pelo menos 1 mês); dependência de álcool, medida através do questionário Cut down, Annoyed by criticism, Guilty and Eye-opener $(\mathrm{CAGE})^{23}$, sendo considerados dependentes alcoólicos aqueles que responderam afirmativamente a duas ou mais perguntas; e índice de massa corporal (IMC), categorizado em $\leq 22,0 \mathrm{~kg} / \mathrm{m}^{2}$ (baixo peso), $22,0-26,9 \mathrm{~kg} / \mathrm{m}^{2}$ (peso adequado), $\geq$ $27,0 \mathrm{~kg} / \mathrm{m}^{2}$ (sobrepeso) ${ }^{24}$. O peso foi medido em balança digital de marca TANITA ${ }^{\circledR}$ (capacidade de 150 kg e precisão de 100 gramas) e a altura foi estimada a partir da medida da altura do joelho (avaliada com estadiômetro infantil, em escala de $100 \mathrm{~cm}$, da marca Indaiá ${ }^{\circledR}$ ) utilizando a equação proposta por Chumlea et al..$^{25}$.

Recebimento de orientações para hábitos saudáveis: foi investigado pela pergunta Desde $<M \hat{E} S>$ do ano passado até agora, algum profissional de saúde orientou o(a) Sr.(a) a ..., substituído pelas seguintes orientações: controlar o peso; reduzir o consumo de sal; reduzir o consumo de açúcar e doces e reduzir o consumo de gorduras $^{5}$. Essas questões foram precedidas por uma pergunta filtro: Desde $<M \hat{E} S>$ do ano passado até agora, o(a) Sr(a). consultou com algum profissional de saúde?, sendo incluídos no estudo somente aqueles que responderam afirmativamente a esta pergunta 5 .

Todas as análises foram realizadas no software Stata versão 12.1 (StataCorp LP, College Station, Estados Unidos) e utilizaram o comando svyset para considerar a complexidade do processo de amostragem e os pesos pós-estratificação. A aplicação do peso pós-estratificação teve o intuito de a igualar a composição sociodemográfica da amostra (sexo e faixa etária) em relação à população idosa elegível para o estudo.

A associação entre as variáveis independentes e os padrões alimentares foi avaliada por meio de regressão logística ordinal, sendo apresentado o odds ratio para estar no tercil de maior adesão de cada padrão alimentar. $\mathrm{O}$ teste Brant foi utilizado para testar a proporcionalidade dos odds.

A análise ajustada foi conduzida em três níveis: no primeiro foram incluídas as variáveis demográficas e socioeconômicas; no segundo, foram adicionadas as variáveis relacionadas à saúde e comportamentos e no terceiro acrescentou-se as orientações recebidas pelos profissionais de saúde. As variáveis referentes às orientações de profissionais de saúde não foram ajustadas entre si, devido a possível colinearidade. Para todas as associações adotou-se um nível de significância de 5\%.

O estudo foi aprovado pelo Comitê de Ética em Pesquisa da Faculdade de Medicina (Universidade Federal de Pelotas) e Certificado de Apresentação para Apreciação Ética (CAAE), em 28 de novembro de 2013. Todos os participantes do estudo assinaram o Termo de Consentimento Livre e Esclarecido (TCLE).

\section{Resultados}

A Tabela 1 apresenta a descrição da amostra em relação às variáveis independentes. A maior parte da amostra foi constituída por mulheres 
$(62,1 \%)$, idosos com idade entre 60 e 69 anos $(59,9 \%)$ e com até sete anos completos de estudo $(67,5 \%)$. A mediana de renda familiar per capita foi de $\mathrm{R} \$ 864,00$ (intervalo interquartil: $\mathrm{R} \$ 666,00-1.500,00)$. Cerca de $13,0 \%$ dos idosos eram fumantes e $1,0 \%$ apresentou dependência alcoólica. A maioria dos idosos tinha sobrepeso $(56,5 \%)$, um terço era hipertenso $(33,8 \%)$ e $23,5 \%$ referiu ter diabetes. As orientações para hábitos saudáveis mais referidas foram: redução do consumo de gordura $(56,7 \%)$, de sal $(56,6 \%)$, e de açúcar (46,3\%).

Dois padrões alimentares foram identificados, os quais explicaram $34,2 \%$ da variabilidade total dos dados de alimentação. Suas características estão descritas na Tabela 2. O padrão denominado "saudável" caracterizou-se pelo consumo de alimentos integrais, frutas, legumes/verduras e

Tabela 1. Descrição da amostra conforme as variáveis demográficas, socioeconômicas, de saúde e comportamentais. Pelotas, RS $(\mathrm{n}=1.426)$.

\begin{tabular}{|c|c|}
\hline Variável & $\%($ IC95\%) \\
\hline \multicolumn{2}{|l|}{ Sexo } \\
\hline Masculino & $37,9(35,7-40,0)$ \\
\hline Feminino & $62,1(60,0-64,3)$ \\
\hline \multicolumn{2}{|l|}{ Idade (anos) } \\
\hline $60-69$ & $59,9(56.9,4-63,0)$ \\
\hline $70-79$ & $23,0(20,9-25,1)$ \\
\hline 80 ou mais & $17,1(14,8-19,3)$ \\
\hline \multicolumn{2}{|l|}{ Escolaridade (anos) } \\
\hline $0-3$ & $36,3(32,4-40,3)$ \\
\hline $4-7$ & $31,2(28,2-34,3)$ \\
\hline $8-11$ & $10,1(8,4-11,8)$ \\
\hline 12 ou mais & $22,3(17,9-26,8)$ \\
\hline \multicolumn{2}{|l|}{ Estado nutricional $^{*}$} \\
\hline Baixo peso & $9,2(7,5-10,9)$ \\
\hline Eutrófico & $34,4(32,1-36,6)$ \\
\hline Sobrepeso & $56,5(53,9-59,0)$ \\
\hline Fumo atual & $13,3(11,3-15,3)$ \\
\hline Dependência de álcool & $1,1(0.5-1,6)$ \\
\hline Hipertensão & $33,8(31,1-36,5)$ \\
\hline Diabetes & $23,5(21,3-25,8)$ \\
\hline \multicolumn{2}{|l|}{$\begin{array}{l}\text { Orientações de profissional de } \\
\text { saúde }\end{array}$} \\
\hline Reduzir o consumo de sal & $56,6(54,0-59,2)$ \\
\hline Reduzir o consumo de açúcar & $46,3(43,6-48,9)$ \\
\hline $\begin{array}{l}\text { Reduzir o consumo de } \\
\text { gordura }\end{array}$ & $56,7(53,9-59,6)$ \\
\hline Reduzir o peso & $36,1(33,5-38,6)$ \\
\hline
\end{tabular}

leite. O padrão "ocidental” foi caracterizado pelo consumo de doces, frituras, alimentos congelados, embutidos e fast food.

A Tabela 3 apresenta a análise bruta e ajustada entre a aderência ao padrão saudável e as variáveis independentes. Após ajuste, os fatores associados à maior adesão ao padrão alimentar saudável foram: sexo feminino, maior idade, maiores níveis de escolaridade e renda e recebimento de orientação para redução de peso e do consumo de açúcar. A menor adesão ao padrão supracitado foi verificada entre idosos tabagistas, dependentes alcoólicos e com baixo peso. Nenhuma associação foi identificada com a presença de morbidades.

A relação entre a adesão ao padrão ocidental e as variáveis independentes encontra-se na Tabela 4. Após ajuste para fatores de confusão, a maior adesão ao padrão ocidental esteve associada ao sexo masculino, maior renda, hábito de fumar, dependência alcoólica e sobrepeso, enquanto que a menor adesão foi encontrada entre aqueles que receberam orientação para a redução do consumo de sal e de açúcar. A presença de morbidades não esteve associada à adesão ao padrão ocidental.

Tabela 2. Distribuição das cargas fatoriais dos padrões de consumo alimentar de idosos. Pelotas, 2014.

\begin{tabular}{lrr}
\hline \multicolumn{1}{c}{$\begin{array}{c}\text { Alimentos / grupos de } \\
\text { alimentos }\end{array}$} & \multicolumn{2}{c}{$\begin{array}{c}\text { Padrões de } \\
\text { consumo alimentar }\end{array}$} \\
\cline { 2 - 3 } & $\begin{array}{c}\text { Padrão } \\
\text { saudável }\end{array}$ & $\begin{array}{c}\text { Padrão } \\
\text { ocidental }\end{array}$ \\
\hline Integrais & $\mathbf{0 , 4 1}$ & $-0,09$ \\
Legumes e verduras & $\mathbf{0 , 5 3}$ & 0,01 \\
Frutas & $\mathbf{0 , 5 5}$ & 0,01 \\
Carne & 0,28 & 0,20 \\
Leite e derivados & $\mathbf{0 , 3 9}$ & $-0,04$ \\
Guloseimas (doces, & $-0,04$ & $\mathbf{0 , 4 2}$ \\
refrigerantes, sucos) & & \\
Frituras & $-0,11$ & $\mathbf{0 , 4 6}$ \\
Conservas, embutidos, & 0,06 & $\mathbf{0 , 5 2}$ \\
enlatados & & \\
Congelados & 0,04 & $\mathbf{0 , 3 7}$ \\
Fast food & 0,02 & $\mathbf{0 , 3 9}$ \\
Número de itens & 4 & 5 \\
\% de explicação da variância & $18,6 \%$ & $15,6 \%$ \\
\% variância acumulada & $18,6 \%$ & $34,2 \%$ \\
\hline Fatores com carga $\geq 0,3$ estão em negrito. & \\
& &
\end{tabular}


Tabela 3. Odds ratio bruto e ajustado da adesão ao padrão saudável segundo características sociodemográficas, comportamentais e de saúde.

\begin{tabular}{|c|c|c|c|c|c|}
\hline \multicolumn{2}{|c|}{ Variável } & $\begin{array}{l}\text { OR bruto } \\
\text { (IC 95\%) }\end{array}$ & \multirow{2}{*}{$\begin{array}{c}\text { Valor-p } \\
<0,001\end{array}$} & $\begin{array}{l}\text { OR ajustado } \\
\text { (IC 95\%) }\end{array}$ & \multirow{2}{*}{$\begin{array}{l}\text { Valor-p } \\
<0,001\end{array}$} \\
\hline Sexo $^{1}$ & Masculino & 1,00 & & 1,00 & \\
\hline & Feminino & $1,59(1,29 ; 1,97)$ & & $1,70(1,35 ; 2,14)$ & \\
\hline Idade $^{1}$ & $60-69$ & 1,00 & $0,022^{\mathrm{t}}$ & 1,00 & $0,003^{t}$ \\
\hline \multirow[t]{2}{*}{ (anos) } & $70-79$ & $1,07(0,85 ; 1,34)$ & & $1,26(0,99 ; 1,61)$ & \\
\hline & 80 ou mais & $1,45(1,07 ; 1,97)$ & & $1,65(1,16 ; 2,35)$ & \\
\hline Escolaridade $^{1}$ & $0-3$ & 1,00 & $<0,001^{\mathrm{t}}$ & 1,00 & $<0,001$ \\
\hline \multirow[t]{3}{*}{ (anos) } & $4-7$ & $1,33(1,02 ; 1,73)$ & & $1,35(1,02 ; 1,79)$ & \\
\hline & $8-11$ & $1,53(1,06 ; 2,20)$ & & $1,35(0,91 ; 2,01)$ & \\
\hline & 12 ou mais & $3,52(2,57 ; 4,81)$ & & $2,20(1,47 ; 3,30)$ & \\
\hline Renda total ${ }^{1}$ & $1^{\circ}($ menor $)$ & 1,00 & $<0,001$ & 1,00 & $<0,001$ \\
\hline \multirow[t]{4}{*}{ (quintis) } & $2^{\circ}$ & $0,93(0,64 ; 1,36)$ & & $0,99(0,68 ; 1,45)$ & \\
\hline & $3^{\circ}$ & $1,14(0,81 ; 1,59)$ & & $1,14(0,81 ; 1,61)$ & \\
\hline & $4^{\circ}$ & $1,93(1,34 ; 2,79)$ & & $1,71(1,16 ; 2,53)$ & \\
\hline & $5^{\circ}$ & $3,84(2,62 ; 5,61)$ & & $2,85(1,78 ; 4,57)$ & \\
\hline \multirow[t]{2}{*}{ Fumo atual ${ }^{2}$} & Não & $2,58(1,91 ; 3,47)$ & $<0,001$ & $2,07(1,492,88)$ & $<0,001$ \\
\hline & Sim & 1,00 & & 1,00 & \\
\hline Dependência de & Não & $4,08(1,42 ; 11,74)$ & 0,006 & $3,93(1,30 ; 11,85)$ & 0,016 \\
\hline álcool$^{2}$ & Sim & 1,00 & & 1,00 & \\
\hline \multirow[t]{3}{*}{ Estado nutricional ${ }^{2}$} & Baixo peso & $0,47(0,33 ; 0,68)$ & $<0,001$ & $0,56(0,38 ; 0,83)$ & 0,016 \\
\hline & Eutrófico & 1,00 & & 1,00 & \\
\hline & Sobrepeso & $0,96(0,76 ; 1,21)$ & & $0,90(0,71 ; 1,15)$ & \\
\hline \multirow[t]{2}{*}{ Hipertensão ${ }^{2}$} & Não & 1,00 & 0,306 & 1,00 & 0,141 \\
\hline & Sim & $1,12(0,90 ; 1,38)$ & & $1,19(0,94 ; 1,50)$ & \\
\hline \multirow[t]{2}{*}{ Diabetes $^{2}$} & Não & 1,00 & 0,452 & 1,00 & 0,554 \\
\hline & Sim & $0,90(0,69 ; 1,18)$ & & $0,91(0,66 ; 1,25)$ & \\
\hline \multicolumn{6}{|c|}{ Orientações de profissional de saúde } \\
\hline Reduzir o consumo & Não & 1,00 & 0,246 & 1,00 & 0,514 \\
\hline de $\mathrm{sal}^{3}$ & Sim & $1,13(0,92 ; 1,40)$ & & $1,08(0,85 ; 1,39)$ & \\
\hline Reduzir o consumo & Não & 1,00 & 0,001 & 1,00 & 0,001 \\
\hline de açúcar ${ }^{3}$ & Sim & $1,44(1,17 ; 1,77)$ & & $1,50(1,19 ; 1,89)$ & \\
\hline Reduzir o consumo & Não & 1,00 & 0,001 & 1,00 & 0,058 \\
\hline de gordura ${ }^{3}$ & Sim & $1,39(1,14 ; 1,70)$ & & $1,24(0,99 ; 1,56)$ & \\
\hline \multirow[t]{2}{*}{ Reduzir o peso ${ }^{3}$} & Não & 1,00 & 0,001 & 1,00 & 0,004 \\
\hline & $\operatorname{Sim}$ & $1,64(1,31 ; 2,05)$ & & $1,47(1,13 ; 1,90)$ & \\
\hline
\end{tabular}

Tendência Linear. ${ }^{1}$ Nível 1: ajuste para as variáveis do mesmo nível; ${ }^{2}$ Nível 2: ajuste para variáveis do mesmo nível e variáveis do nível 1; ${ }^{3}$ Nível 3: ajuste para variáveis do nível 1 e 2. Nota: um odds ratio acima da unidade indica maior chance de estar em no tercil de maior adesão ao padrão, enquanto que um odds ratio abaixo da unidade indica menor chance de aderir ao padrão.

\section{Discussão}

Este estudo identificou dois padrões alimentares entre os idosos: o "padrão saudável” e o "padrão ocidental”. Mulheres, indivíduos não fumantes e sem dependência alcoólica aderiam mais ao padrão saudável, enquanto que homens, indivíduos fumantes, dependentes alcóolicos e com sobrepeso aderiam mais ao padrão ocidental. Maior renda e escolaridade estiveram associadas à maior adesão de ambos os padrões alimentares. O recebimento de orientações para hábitos saudáveis esteve associado à maior adesão ao padrão saudável e menor adesão ao padrão ocidental. As DCNT investigadas (diabetes e hipertensão) não estiveram associadas à adesão aos padrões alimentares identificados.

Diferentemente do observado por Ferreira et al. ${ }^{10}$, neste estudo, maior renda esteve associada à maior adesão tanto ao padrão saudável quanto 
Tabela 4. Odds ratio bruto e ajustado da adesão ao padrão ocidental segundo características sociodemográficas, comportamentais e de saúde.

\begin{tabular}{|c|c|c|c|c|c|}
\hline \multicolumn{2}{|c|}{ Variável } & \multirow{2}{*}{$\begin{array}{c}\begin{array}{c}\text { OR bruto } \\
\text { (IC 95\%) }\end{array} \\
1,69(1,38 ; 2,06)\end{array}$} & \multirow{2}{*}{$\begin{array}{c}\text { Valor-p } \\
<0,001\end{array}$} & \multirow{2}{*}{$\begin{array}{c}\begin{array}{c}\text { OR ajustado } \\
\text { (IC 95\%) }\end{array} \\
1,72(1,41 ; 2,10)\end{array}$} & \multirow{2}{*}{$\begin{array}{l}\text { Valor-p } \\
<0,001\end{array}$} \\
\hline Sexo $^{1}$ & Masculino & & & & \\
\hline & Feminino & 1,00 & & 1,00 & \\
\hline Idade $^{1}$ & $60-69$ & $1,36(0,99 ; 1,86)$ & $0,019^{t}$ & $1,26(0,90 ; 1,76)$ & 0,117 \\
\hline \multirow[t]{2}{*}{ (anos) } & $70-79$ & $1,03(0,73 ; 1,45)$ & & $1,00(0,70 ; 1,44)$ & \\
\hline & 80 ou mais & 1,00 & & 1,00 & \\
\hline Escolaridade ${ }^{1}$ & $0-3$ & 1,00 & $0,002^{\mathrm{t}}$ & 1,00 & 0,666 \\
\hline \multirow[t]{3}{*}{ (anos) } & $4-7$ & $1,14(0,88 ; 1,46)$ & & $0,97(0,74 ; 1,26)$ & \\
\hline & $8-11$ & $1,39(0,94 ; 2,06)$ & & $1,19(0,77 ; 1,85)$ & \\
\hline & 12 ou mais & $1,51(1,14 ; 1,99)$ & & $1,17(0,82 ; 1,67)$ & \\
\hline Renda total ${ }^{1}$ & $1^{\circ}($ menor $)$ & 1,00 & $<0,001$ & 1,00 & $<0,001$ \\
\hline \multirow[t]{4}{*}{ (quintis) } & $2^{\circ}$ & $0,97(0,69 ; 1,37)$ & & $0,95(0,67 ; 1,34)$ & \\
\hline & $3^{\circ}$ & $1,31(0,93 ; 1,85)$ & & $1,26(0,89 ; 1,78)$ & \\
\hline & $4^{\circ}$ & $1,72(1,29 ; 2,29)$ & & $1,62(1,18 ; 2,20)$ & \\
\hline & $5^{\circ}$ & $1,87(1,29 ; 2,71)$ & & $1,65(1,12 ; 2,42)$ & \\
\hline \multirow[t]{2}{*}{ Fumo atual ${ }^{2}$} & Não & 1,00 & 0,001 & 1,00 & 0,007 \\
\hline & Sim & $1,58(1,20 ; 2,07)$ & & $1,51(1,12 ; 2,04)$ & \\
\hline Dependência de & Não & 1,00 & 0,002 & 1,00 & 0,018 \\
\hline álcool$^{2}$ & Sim & $4,51(1,77 ; 11,41)$ & & $2,86(1,20 ; 6,81)$ & \\
\hline \multirow[t]{3}{*}{ Estado nutricional ${ }^{2}$} & Baixo peso & $1,14(0,78 ; 1,66)$ & 0,126 & $1,15(0,77 ; 1,74)$ & 0,023 \\
\hline & Eutrófico & 1,00 & & 1,00 & \\
\hline & Sobrepeso & $1,24(1,01 ; 1,52)$ & & $1,35(1,09 ; 1,67)$ & \\
\hline \multirow[t]{2}{*}{ Hipertensão² } & Não & 1,00 & 0,096 & 1,00 & 0,119 \\
\hline & Sim & $0,84(0,69 ; 1,03)$ & & $0,83(0,66 ; 1,05)$ & \\
\hline \multirow[t]{2}{*}{ Diabetes $^{2}$} & Não & 1,00 & 0,316 & 1,00 & 0,263 \\
\hline & Sim & $0,89(0,70 ; 1,12)$ & & $0,87(0,69 ; 1,11)$ & \\
\hline \multicolumn{6}{|c|}{ Orientações de profissional de saúde } \\
\hline Reduzir o consumo & Não & 1,00 & 0,001 & 1,00 & 0,045 \\
\hline de $\mathrm{sal}^{3}$ & Sim & $0,71(0,59 ; 0,87)$ & & $0,78(0,61 ; 0,99)$ & \\
\hline Reduzir o consumo & Não & 1,00 & 0,003 & 1,00 & 0,008 \\
\hline de açúcar ${ }^{3}$ & Sim & $0,75(0,62 ; 0,90)$ & & $0,74(0,59 ; 0,92)$ & \\
\hline Reduzir o consumo & Não & 1,00 & 0,055 & 1,00 & 0,249 \\
\hline de gordura ${ }^{3}$ & Sim & $0,82(0,66 ; 1,00)$ & & $0,87(0,69 ; 1,10)$ & \\
\hline \multirow[t]{2}{*}{ Reduzir o peso ${ }^{3}$} & Não & 1,00 & 0,756 & 1,00 & 0,736 \\
\hline & Sim & $1,03(0,84 ; 1,28)$ & & $0,96(0,76 ; 1,21)$ & \\
\hline
\end{tabular}

'Tendência linear. ${ }^{\sharp}$ Nível 1: ajuste para as variáveis do mesmo nível; Nível 2: ajuste para variáveis do mesmo nível e variáveis do nível 1; Nível 3: ajuste para variáveis do nível 1 e 2. Nota: um odds ratio acima da unidade indica maior chance de estar em no tercil de maior adesão ao padrão, enquanto que um odds ratio abaixo da unidade indica menor chance de aderir ao padrão.

ao ocidental. Embora maior renda facilite a aquisição de alimentos saudáveis, normalmente mais caros, ela está associada ao acesso a alimentos de uma forma geral, incluindo aqueles não saudáveis, o que pode explicar a associação com ambos padrões alimentares ${ }^{26}$. Por outro lado, o nível educacional esteve associado somente à maior adesão ao padrão alimentar saudável, como previamente observado ${ }^{10}$. Indivíduos mais es- colarizados possivelmente valorizam mais a importância de uma alimentação saudável, muitas vezes como forma de prevenção ou controle das DCNT, refletindo em maior adesão a comportamentos saudáveis, como o consumo de alimentos integrais, legumes e frutas.

Assim como em outros estudos, mulheres aderiram mais ao padrão alimentar saudável ${ }^{10,12}$. No geral, as mulheres apresentam um perfil mais 
voltado para atenção à saúde, possuem maior cuidado em relação à alimentação do que homens, estando mais engajadas em dietas para a redução de peso e maior qualidade de vida, preocupando-se mais com a qualidade dos alimentos consumidos e buscando um maior equilíbrio alimentar ${ }^{12}$.

Idosos com baixo peso aderiram menos ao padrão alimentar saudável quando comparados aos eutróficos. Estes podem apresentar outros problemas de saúde que comprometem a capacidade de alimentar-se de forma adequada. Nesta mesma população, homens idosos com baixo peso apresentaram maior dependência para atividades relacionadas à alimentação, como compra ou preparo de alimentos, embora o mesmo padrão não tenha sido observado nas mulheres ${ }^{27}$. Por outro lado, idosos com sobrepeso aderiram mais ao padrão ocidental. $\mathrm{O}$ estudo de Ledikwe et al. ${ }^{28}$, realizado na zona rural dos Estados Unidos, mostrou que idosos com um padrão alimentar caracterizado pelo maior consumo de pão, carnes processadas, ovos, gordura e doces, tinham o dobro de chance de serem obesos ou de terem uma circunferência da cintura acima dos pontos de corte identificados como fatores de risco para obesidade.

Idosos que não fumavam apresentaram maior chance de aderirem ao padrão saudável, enquanto que os tabagistas apresentaram maior chance de aderirem ao padrão ocidental. Em populações mais jovens foram encontrados resultados semelhantes, com tabagistas apresentando menor consumo de frutas, vegetais e produtos lácte$\mathrm{os}^{29,30}$. Assumpção et al. ${ }^{31}$, utilizando o Índice de Qualidade da Dieta Revisado (IQD-R), também mostraram que idosos tabagistas tinham uma dieta de pior qualidade. É provável que indivíduos tabagistas tenham menor consideração por sua saúde do que não fumantes ou ex-fumantes, o que pode resultar na escolha de uma dieta de menor qualidade ${ }^{32}$.

Idosos não dependentes alcoólicos tiveram maior chance de aderirem ao padrão saudável comparados àqueles dependentes de álcool. Tal achado corrobora estudos prévios, que mostraram que o aumento da frequência de consumo do álcool se associa à pior qualidade da dieta ${ }^{31,33,34}$. Esta associação entre pior qualidade da dieta e consumo de álcool pode ser explicada pelo elevado conteúdo energético das bebidas alcoólicas, o que acaba por diminuir o apetite, e pelos tipos de alimentos que geralmente são escolhidos para acompanhar o seu consumo ${ }^{33}$.

O recebimento de orientação para redução do consumo de açúcar e do peso esteve associa- do à maior adesão ao padrão saudável, enquanto que o recebimento de orientação para redução do consumo de sal e açúcar esteve associado à menor adesão ao padrão ocidental. Esse achado permite identificar que essas orientações influenciam positivamente o consumo alimentar de idosos, direcionando-os para um padrão alimentar mais saudável. Todavia, a orientação para a redução de gordura não esteve associada à menor adesão ao padrão ocidental. Os alimentos que compõem o padrão ocidental são, em sua maioria, alimentos processados e pode ser que os idosos não associem esses alimentos ao maior consumo de gordura. Dessa forma, também é importante que as orientações prestadas sejam suficientemente claras para que possam atingir seus objetivos, especialmente se tratando da população idosa.

O sucesso do tratamento não medicamentoso de algumas DCNT, como diabetes e hipertensão, depende da adoção de um plano alimentar saudável $^{35,36}$. Idosos portadores de DCNT são aqueles que mais utilizam os serviços de saúde para o monitoramento de suas condições e redução dos agravos gerados por essas doenças ${ }^{37}$ e também os que mais frequentemente recebem orientações para hábitos saudáveis ${ }^{5}$. Estudos prévios realizados avaliando marcadores de alimentação saudável e não saudável em adultos e idosos portadores de hipertensão e/ou diabetes, demonstraram que, em geral, estes pacientes tendem a apresentar indicadores de consumo alimentar satisfatório para a maioria das recomendações ${ }^{16,38}$. Todavia, neste estudo, a adesão aos padrões alimentares foi bastante semelhante entre portadores de diabetes e hipertensão e aqueles sem essas enfermidades.

Este estudo aponta diferentes características associadas ao padrão alimentar de idosos em Pelotas, no Sul do Brasil. Estudos que avaliam os padrões alimentares nesta população ainda são escassos e, no Brasil, até o presente momento, apenas dois estudos foram identificados, os quais não utilizaram uma amostra populacional representativa $^{10,18}$.

Algumas limitações do presente estudo precisam ser pontuadas. O questionário de frequência alimentar utilizado, por ser reduzido, não permite um amplo panorama dos alimentos consumidos por idosos. Além disso, as informações de consumo referem-se à semana anterior à entrevista, não refletindo necessariamente a variabilidade de consumo alimentar dos idosos. Entretanto, este período foi escolhido de forma a minimizar o viés de recordatório, que pode ser comum, especialmente em idosos, e identificar marcadores de consumo saudável e não saudável. 
A extração dos padrões alimentares por meio de análise de componentes principais permite com que se conheça o comportamento alimentar de uma população, mas dificilmente serão replicáveis em outros estudos, dificultando a comparabilidade dos padrões obtidos. Além disso, embora os indivíduos apresentem uma maior adesão a um determinado padrão, os fatores obtidos nesta análise não são excludentes. A perda diferencial da amostra com relação à idade também é uma limitação do estudo, embora não afete as principais conclusões do estudo. Uma vez que a adesão ao padrão saudável foi menor nos idosos mais jovens, é esperado que a estimativa de efeito reportada na associação entre essas variáveis possa estar apenas subestimada.

Existe, ainda, a possibilidade de causalidade reversa na associação entre padrões alimentares e estado nutricional, tabagismo e dependência alcoólica. No entanto, cabe salientar que o objetivo deste estudo foi avaliar fatores relacionados à adesão dos padrões alimentares e não determinar relações causais. Além disso, mesmo que as variáveis tenham sido coletadas ao mesmo momento, todas incluíam algum critério de temporalidade prévia, reduzindo a chance de viés.

\section{Conclusão}

Maior adesão ao padrão alimentar saudável foi observada em mulheres, idosos mais escolarizados, não tabagistas e sem dependência alcoólica, enquanto que maior adesão ao padrão ocidental foi encontrada em indivíduos do sexo masculino, tabagistas, dependentes alcoólicos e naqueles com sobrepeso. Estratégias visando promover uma alimentação saudável em idosos devem priorizar indivíduos com estas características (homens, tabagistas, com consumo elevado de álcool e com sobrepeso), uma vez que configuram um grupo de maior risco para o consumo de uma dieta constituída principalmente por alimentos processados e ultraprocessados, que pode refletir no agravamento do estado nutricional e de outras condições de saúde, como DCNT, comuns nesta faixa etária. Foi observado que a maior renda se associou à maior adesão de ambos os padrões alimentares (saudável e ocidental), reafirmando a importância de ações educativas relativas à alimentação em todos os seguimentos sociais, uma vez que a escolaridade, independente da renda, parece ter efeito positivo do consumo alimentar. Nesse sentido, há necessidade do fortalecimento de orientações sobre hábitos saudáveis nos serviços de saúde de forma a promover cada vez mais práticas saudáveis, como alimentação adequada. A orientação sobre hábitos saudáveis é uma medida sem custo que pode ser realizada em qualquer contato dos profissionais com seus pacientes. 


\section{Colaboradores}

AP Gomes participou da concepção e planejamento do estudo, análise e interpretação dos dados e redação do manuscrito. ALG Soares, IO Bierhals, LS Vieira e TR Flores colaboraram na interpretação dos resultados e na redação do manuscrito. MCF Assunção e H Gonçalves colaboraram na interpretação dos dados e revisão crítica no conteúdo.

\section{Agradecimentos}

Esta pesquisa contou com o apoio financeiro da Coordenação de Aperfeiçoamento de Pessoal de Nível Superior (CAPES), com recursos provenientes do Programa de Excelência Acadêmica (PROEX).

\section{Referências}

1. Instituto Brasileiro de Geografia e Estatística (IBGE). Pesquisa nacional por amostra de domicílios: síntese de indicadores 2015. Rio de Janeiro: IBGE; 2016.

2. Instituto Brasileiro de Geografia e Estatística (IBGE). Síntese de indicadores sociais: uma análise das condições de vida da população brasileira: 2016. Rio de Janeiro: IBGE; 2016.

3. Costa CdS, Schneider BC, Cesar JA. Obesidade geral e abdominal em idosos do Sul do Brasil: resultados do estudo COMO VAI? Cien Saude Colet 2016; 21(11):3585-3596.

4. Instituto Brasileiro de Geografia e Estatística (IBGE). Pesquisa Nacional por Amostra de Domicílios (PNAD). Um panorama da saúde no Brasil. Acesso e Utilização dos serviços, condições de saúde e fatores de risco e proteção à saúde. Rio de Janeiro: IBGE; 2010.

5. Flores TR, Nunes BP, Assunção MCF, Bertoldi AD. Hábitos saudáveis: que tipo de orientação a população idosa está recebendo dos profissionais de saúde? Revista Brasileira de Epidemiologia 2016; 19(1):167180.

6. Brasil. Ministério da Saúde (MS). Política Nacional de Promoção da Saúde. Brasília: MS; 2010.

7. Azevedo ECC, Diniz AS, Monteiro JS, Cabral PC. Padrão alimentar de risco para as doenças crônicas não transmissíveis e sua associação com a gordura corporal - uma revisão sistemática. Cien Saude Colet 2014; 19(5):1447-1458.

8. Carvalho CA, Fonsêca PCA, Nobre LN, Priore SE, Franceschini SCC. Metodologias de identificação de padrões alimentares a posteriori em crianças brasileiras: revisão sistemática. Cien Saude Colet 2016; 21(1):143-154.

9. World Health Organization (WHO). Preparation and use of food based dietary guidelines. Geneva: WHO; 1998. [Report of a Joint FAO/ WHO Consultation]

10. Ferreira PM, Papini SJ, Corrente JE. Diversity of eating patterns in older adults: A new scenario? Revista de Nutrição 2014; 27(1):67-79.

11. Brasil. Ministério do Desenvolvimento Social e Combate à Fome (MDS). Marco de referência de educação alimentar e nutricional para as políticas públicas. Brasília: MDS; 2012.

12. Gomes AP, Soares ALG, Gonçalves H. Baixa qualidade da dieta de idosos: estudo de base populacional no sul do Brasil. Cien Saude Colet 2016; 21(11):3417-3428.

13. Walker RL, Gee ME, Bancej C, Nolan RP, Kaczorowski J, Joffres M, Bienek A, Gwadry-Sridhar F, Campbell NR. Health behaviour advice from health professionals to Canadian adults with hypertension: results from a national survey. Can J Cardiol 2011; 27(4):446454.

14. Agborsangaya CB, Gee ME, Johnson ST, Dunbar P, Langlois M-F, Leiter LA, Pelletier C, Johnson JA. Determinants of lifestyle behavior in type 2 diabetes: results of the 2011 cross-sectional survey on living with chronic diseases in Canada. BMC Public Health 2013; 13:451.

15. Lopes ACS, Toledo MTT, Câmara AMCS, Menzel H-JK, Santos LC. Condições de saúde e aconselhamento sobre alimentação e atividade física na Atenção Primária à Saúde de Belo Horizonte-MG. Epidemiologia e Serviços de Saúde 2014; 23(3):305-316. 
16. Destri K, Zanini RdV, Assunção MCF. Prevalência de consumo alimentar entre hipertensos e diabéticos na cidade de Nova Boa Vista, Rio Grande do Sul, Brasil, 2013. Epidemiologia e Serviços de Saúde 2017; 26(4):857-868.

17. Flores TR, Gomes AP, Soares ALG, Nunes BP, Assunção MCF, Gonçalves H, Bertoldi AD. Aconselhamento por profissionais de saúde e comportamentos saudáveis entre idosos: estudo de base populacional em Pelotas, sul do Brasil, 2014. Epidemiologia e Serviços de Saúde 2018; 27(1):e201720112.

18. Souza JD, Martins MV, Franco FS, Martinho KO, Tinôco AL. Dietary patterns of the elderly: characteristics and association with socioeconomic aspects. Revista Brasileira de Geriatria e Gerontologia 2016; 19(6):970-977.

19. Ferreira-Nunes PM, Corrente JE, Papini SJ. Padrões alimentares e ingestão de nutrientes em idosos: análise com diferentes abordagens metodológicas. Cien Saude Colet 2018; 23(12):4085-4094.

20. Brasil. Ministério da Saúde (MS). Guia alimentar para a população brasileira: promovendo a alimentação saudável. Brasília: MS; 2008.

21. Brasil. Ministério da Saúde (MS). Guia alimentar para a população brasileira. 2a ed. Brasília: MS; 2014.

22. Olinto MTA. Padrões alimentares: análise de componentes principais. In: Kac G, Sichieri R, Gigante DP, organizadores. Epidemiologia nutricional. Rio de Janeiro: Editora Fiocruz, Atheneu; 2007. p. 213-225.

23. Ewing JA. Detecting alcoholism. The CAGE questionnaire. JAMA 1984; 252(14):1905-1907.

24. Brasil. Ministério da Saúde (MS). Orientações para a coleta e análise de dados antropométricos em serviços de saúde: Norma Técnica do Sistema de Vigilância Alimentar e Nutricional - SISVAN. Brasília: MS; 2011.

25. Chumlea WMC, Guo SS, Roche AF, Steinbaugh ML. Prediction of body weight for the nonambulatory elderly from anthropometry. J Am Diet Assoc 1988; 88(5):564-8.

26. Instituto Brasileiro de Geografia e Estatística (IBGE). Pesquisa de Orçamentos Familiares 2008-2009 - POF. Rio de Janeiro: IBGE; 2010.

27. Bierhals IO, Meller FO, Assunção MCF. Dependência para a realização de atividades relacionadas à alimentação em idosos. Cien Saude Colet 2016; 21(4):12971308.

28. Ledikwe JH, Smiciklas-Wright H, Mitchell DC, Miller CK, Jensen GL. Dietary patterns of rural older adults are associated with weight and nutritional status. J Am Geriatr Soc 2004; 52(4):589-595.

29. Wilson DB, Smith BN, Speizer IS, Bean MK, Mitchell KS, Uguy LS, Fries EA. Differences in food intake and exercise by smoking status in adolescents. Prev Med 2005; 40(6):872-879.
30. Baer Wilson D, Nietert PJ. Patterns of fruit, vegetable, and milk consumption among smoking and nonsmoking female teens. Am J Prev Med 2002; 22(4):240246.

31. Assumpção D, Domene SMA, Fisberg RM, Barros MBA. Qualidade da dieta e fatores associados entre idosos: estudo de base populacional em Campinas, São Paulo, Brasil. Cad Saude Publica 2014;30(8):16801694.

32. Cade JE, Margetts BM. Relationship between diet and smoking - is the diet of smokers different? J Epidemiol Community Health 1991; 45(4):270-272.

33. Breslow RA, Guenther PM, Juan W, Graubard BI. Alcoholic beverage consumption, nutrient intakes, and diet quality in the US adult population, 1999-2006. J Am Diet Assoc 2010; 110(4):551-562.

34. Herbeth B, Samara A, Stathopoulou M, Siest G, Visvikis-Siest S. Alcohol Consumption, Beverage Preference, and Diet in Middle-Aged Men from the STANISLAS Study. J Nutr Metab 2012; 2012:987243.

35. Malachias M, Franco R, Forjaz C, Pierin A, Gowdak M, Klein M, Matsudo V. 7th Brazilian Guideline of Arterial Hypertension: Chapter 6 - Non-pharmacological treatment. Arq Bras Cardiol 2016; 107(3 Supl. 3):30-34.

36. Sociedade Brasileira de Diabetes. Diretrizes da Sociedade Brasileira de Diabetes 2015-2016. São Paulo: AC Farmacêutica; 2016.

37. Lima-Costa MF, Loyola Filho AId, Matos DL. Tendências nas condições de saúde e uso de serviços de saúde entre idosos brasileiros: um estudo baseado na Pesquisa Nacional por Amostra de Domicílios (1998, 2003). Cad Saude Publica 2007; 23(10):2467-2478.

38. Zanchim MC, Kirsten VR, De Marchi ACB. Marcadores do consumo alimentar de pacientes diabéticos avaliados por meio de um aplicativo móvel. Cien Saude Colet 2018; 23(12):4199-4208.

Artigo apresentado em 28/09/2017

Aprovado em 11/10/2018

Versão final apresentada em 13/10/2018 